\title{
Study of the effects of kinesthetic motor imagery in patients with heart failure
}

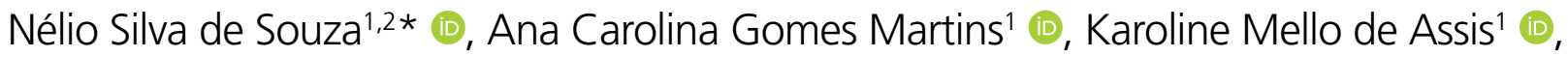 \\ Lúcia Brandão de Oliveira' ${ }^{1}$ (), Rosiane Fátima Silveira de Abreu ${ }^{1}$ (1), \\ Marco Antônio Araújo-Leite ${ }^{\circledR}$, Marco Antônio Orsini Neves $2,3,4,5$ (), \\ Nicolle dos Santos Moraes Nunes ${ }^{3}$ (D) Victor Hugo do Vale Bastos ${ }^{5}$ (D), \\ Júlio Guilherme Silva ${ }^{3,6}$ [D, Alba Barros Souza Fernandes ${ }^{1}$
}

\section{SUMMARY}

OBJECTIVE: The aim was to investigate the acute effect of kinesthetic motor imagery of the 2-minute walk test on hemodynamic and cardiopulmonary variables in patients with heart failure.

METHODS: Twenty participants were recruited for the analysis of these variables before and after the execution and imagination of the 2-minute walk test, with the number of laps executed and imagined being recorded.

RESULTS: The main results observed showed that (1) there was no difference in the number of laps executed and imagined ( $p=0.41$ ), indicating that the participants actually imagined the test and (2) the motor imagery of the 2-minute walk test immediately increased $(p<0.001)$ the heart and respiratory rates.

CONCLUSION: The motor imagery seems to have acute effects on the cardiopulmonary anticipatory responses of a patient with heart failure. KEYWORDS: Heart failure. Imagery, psychotherapy. Heart rate. Respiratory frequency. Autonomic nervous system.

\section{INTRODUCTION}

Heart failure (HF) is defined as a complex clinical syndrome, characterized by functional or structural injury that compromises the ventricular filling or its ejection fraction ${ }^{1}$. These changes promote a decrease in the metabolic support of the tissue, resulting in a set of signs and symptoms, such as dyspnea, fatigue, and water retention ${ }^{1,2}$. The HF is categorized by the New York Heart Association (NYHA), according to the severity of symptoms related to the individual, in degrees of variation from I to IV, according to the functional impairment ${ }^{2}$. In this context, some patients with HF may have low tolerance to exercise associated with marked metabolic and/or respiratory responses. This condition can cause inactivity persistently and a consequent decrease in cardiopulmonary function ${ }^{1,3}$. Cardiopulmonary physiotherapy is highly evidenced and recommended for the treatment of patients with $\mathrm{HF}^{2,3}$. However, some of these patients may have low tolerance to the exercises proposed in conventional physiotherapy ${ }^{1-3}$.

Motor imagery (MI) is defined as the ability to mentally simulate a movement, without actually executing $\mathrm{it}^{4,5}$. The execution and imagination of the same movement are related phenomena and have an entirely voluntary motor control profile ${ }^{6}$. Different studies have shown that the temporal and biomechanical similarities between the execution and imagination of the same task ${ }^{4,5}$, such as the time required for an individual to walk and imagine that he/she is walking the same fixed

\footnotetext{
${ }^{1}$ Centro Universitário Serra dos Órgãos, Department of Physical Therapy - Teresópolis (RJ), Brazil.

¿Universidade Federal Fluminense, Department of Neurology and Neuroscience - Niterói (RJ), Brazil.

${ }^{3}$ Universidade Iguaçu, Department of Medicine - Nova Iguaçu (RJ), Brazil.

${ }^{4}$ Universidade de Vassouras, Department of Medicine - Vassouras (RJ), Brazil.

${ }^{5}$ Universidade Federal do Delta do Parnaíba, Department of Brain Mapping and Functionality Lab - Parnaíba (PI), Brazil.

${ }^{6}$ Universidade Federal do Rio de Janeiro, Department of Physical Therapy - Rio de Janeiro (RJ), Brazil.

*Corresponding author: neliosds@gmail.com/neliosouza@unifeso.edu.br

Conflicts of interest: the authors declare there is no conflicts of interest. Funding: none.

Received on January 31, 2021. Accepted on March 09, 2021.
} 
distance, are similar (isochronous principle) without statistical difference $^{6}$. The MI presents two distinct strategies: visual and kinesthetic. Specifically, in the kinesthetic MI, the individual "feels" performing the imagined movement ${ }^{4,5}$, accessing somato-motor areas related to the perception of movement and part of the circuits related to the preparation and programming of the action ${ }^{7}$.

Traditionally, the autonomic nervous system (ANS) regulates different visceral functions at the unconscious level. However, conscious modulations using kinesthetic MI are able to exert unconscious changes at the ANS level in healthy individuals or athletes, leading to, for example, an increase in heart rate (HR) and respiratory frequency $(\mathrm{RF})^{8-10}$. These modulations occur due to the similarities in the brain areas that are responsible for the preparation and programming of the same task (dorsolateral prefrontal cortex, hypothalamus, cerebellum, basal ganglia, reticular formation, and supplementary motor area $)^{11}$. Thus, these brain areas participate in the control of anticipatory cardiopulmonary responses (feed forward) ${ }^{11}$ during both $\mathrm{MI}^{12}$ and the execution of a physical activity ${ }^{13}$. Recently, our group published an editorial suggesting that some NYHA functional classes, which did not tolerate exercise, could benefit from the use of MI ${ }^{14}$. However, the effects of MI on HF patients are not yet known. Our hypothesis is that MI can modulate cardiopulmonary variables in HF patients in a way similar to healthy individuals. In this context, the aim of this study was to investigate the acute effect of kinesthetic MI of the 2-minute walk test (2MWT) on the hemodynamic and cardiopulmonary variables of patients with HF.

\section{METHODS}

\section{Sample}

Twenty participants were recruited with clinical and echocardiographic diagnosis of $\mathrm{HF}$ with preserved or reduced ejection fraction, in NYHA functional class II, as they have the tolerance to perform the test. All patients were followed up regularly at the outpatient clinic of the Clínica de Insuficiência Cardíaca (CLIC) of the Centro Universitário Serra dos Órgãos (UNFESO).

\section{Ethics and consent form}

The protocol was forwarded and approved by the Research Ethics Committee of UNIFESO through Brazil Platform (seem $\left.n^{\circ} .2,764,323\right)$. All participants signed a Free and Clarified Consent Term, in accordance with the resolution CNS 466/12 and the ethical standards established in the Declaration of Helsinki (2008).

\section{Inclusion and exclusion criteria}

This study included individuals diagnosed with HF in NYHA functional class II of both genders. Individuals with any limitation to walking and cognitive deficit in the Mini Mental State Exam (MMSE) were excluded ${ }^{15}$.

\section{Questionnaires}

The Duke Activity Status Index (DASI) was used to assess the functional capacity profile of the research participants ${ }^{16}$. The volunteers' ability and vividness to perform MI were assessed using the Kinesthetic and Visual Imagery Questionnaire (KVIQ-10) ${ }^{17}$.

\section{Data collection strategies}

The respiratory muscle strength was assessed by means of an analog and calibrated manovacuometer. The peak expiratory flow was quantified by the Peak Flow Meter ${ }^{\circledR}$. Thoracic cirtometry was performed, and diameters were recorded at rest, maximum inspiration, and expiration. For the measurement of blood pressure (BP), a properly calibrated MISSOURI ${ }^{\circledast}$ sphygmomanometer was used and the peripheral oxygen saturation was verified with the Bioland ${ }^{\circledR}$ portable pulse oximeter. Experimental protocol

Initially, the MMSE ${ }^{15}$, DASI ${ }^{16}$, and KVIQ- $10^{17}$ questionnaires were applied. Then, the patients performed the $2 \mathrm{MWT}^{18}$. After performing the 2MWT, the participants were asked to sit in a chair for $10 \mathrm{~min}$ so that their cardiorespiratory parameters returned to the values close to the parameters at rest, establishing a baseline. Subsequently, the kinesthetic MI of the 2MWT was performed, with the patient sitting in a chair facing the track where the test was performed with his/her eyes closed and hands resting on his/her thighs. All participants were instructed to perform MI through the following instruction: "imagine that you are walking as you just did. You should feel like you are going as far as possible until you hear the end warning." The participants were instructed to count the number of completed laps during two min of the test (TC2M) executed and imagined with a digital numerical counter.

The variables recorded before and after the execution and imagination of the TC2M were RF, HR, recovery HR in the first minute (RHR1), systolic blood pressure (SBP), diastolic blood pressure (DBP), mean arterial pressure (MAP), pulse pressure (PP), peripheral oxygen saturation $\left(\mathrm{SpO}_{2}\right)$, subjective dyspnea scale (SDS), and fatigue using the Modified Borg Scale. The RF was recorded by the examiner, and HR was obtained by using a Polar FT1 ${ }^{\circledR}$ frequency meter.

\section{Data analysis}

The normality of the data was previously verified using the Shapiro-Wilk test. The effect size $(d)$ was estimated by partial etasquared $\left(\eta_{p}^{2}\right)$ and the statistical power in $80 \%$. The hemodynamic 
and cardiorespiratory parameters, evaluated before and after the execution and imagination of the 2MWT, were compared using the repeated analysis of variance (ANOVA) followed by the Bonferroni post-test. The paired Student's $t$-test was used to compare the number of runs executed and imaged on the 2MWT, as well as the other variables analyzed. The Spearman's correlation test was used to verify the degree of association between the cardiopulmonary variables after the MI. All the analyses were performed using the Statistical Package for the Social Sciences (SPSS, version 20), assuming an alpha significance level of $\mathrm{p} £ 0.05$.

\section{RESULTS}

\section{Sample characteristics}

The following values are presented as mean \pm standard deviation (minimum-maximum). Ten volunteers of both sexes $(\mathrm{n}=20)$ were selected with the age of 59.1 \pm 7.5 (46-75) years and a body mass index (BMI) of 29.2 \pm 6.0 (23-44). Different levels of education were reported, with at least the completed fourth grade and at the most completed higher education. In the examination for cognitive impairment screening by the MMSE, the participants presented $24.7 \pm 2.0$ (21-28). In general, the volunteers presented a functional capacity profile moderated by the DASI Questionnaire with $27.86 \pm 13.38(9.95-50.95)$ points, which allowed them to participate in the proposed protocol.

\section{Objective evaluation}

On physical examination, none of the participants showed changes in HR or pulmonary auscultation. The following parameters were assessed at the start and end of the protocol: thoracic cirtometry, respiratory muscle strength, peak expiratory flow, $\mathrm{SBP}, \mathrm{DBP}, \mathrm{SpO}_{2}$, Borg fatigue, and SDS. All these variables showed no statistically significant difference $(\mathrm{p}>0.05)$.

\section{Comparisons between TC2M execution and imagination}

The main results showed that:

(1) the comparison between the number of executed [ $6 \pm 1.25$ (4-8)] and imagined [7.8 $\pm 2.74(4-11)]$ laps was not statistically different $[\mathrm{t}(9)=-2.37 ; \mathrm{p}=0.41 ; \mathrm{d}=1.21]$, indicating similarity and suggesting that the participants actually imagined the proposed task (isochronic principle);

(2) the vividness shown by the participants on the KVIQ-10 Scale reported after the MI of the $2 \mathrm{MWT}$ was considered high [3.9 $\pm 0.8(3-5)]$;

(3) there was an increase in HR after MI of the $2 \mathrm{MWT}$ when compared with the moments before (69 \pm 7.9$)$ and after $(80 \pm 11.1)$, with a statistically significant difference
$\left[F(2.49)=45.0 ; \mathrm{p}<0.001 ; \eta^{2}=0.89 ;\right.$ power $=100 \%$; Figur e 1]. Figure 2 shows the HR behavior of the participants before, during, and after the MI of the 2MWT; and

(4) there was also an increase in the RF after the MI of the 2MWT when compared with the moments before (16.1 \pm 2.0$)$ and after (20.1 \pm 2.44$)$, with a significant difference [Friedman $\chi^{2}(3)=1.22 ; \mathrm{p}<0.001 ; \eta_{\mathrm{p}}^{2}=0.51$; power $=100 \%$; Figure 3].

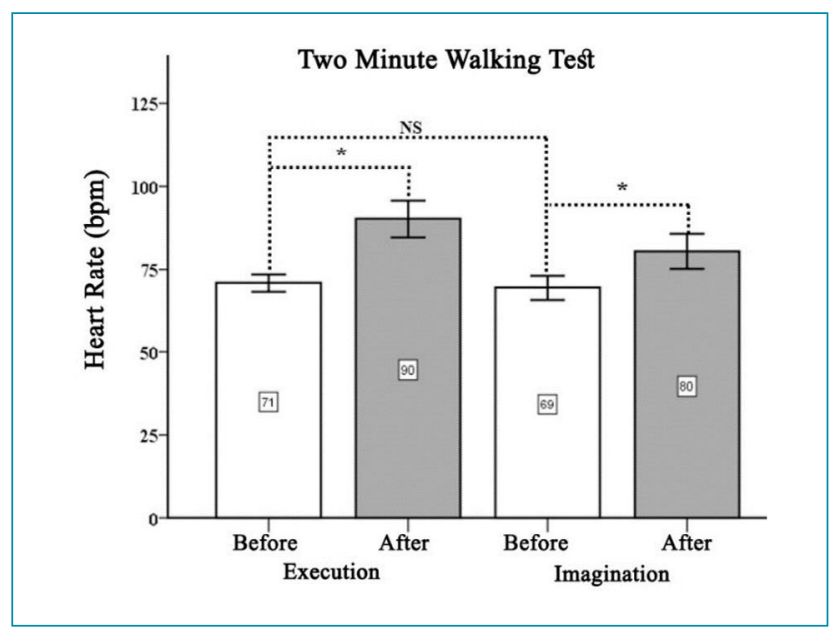

Figure 1. The participants $(n=20)$ showed an increase in heart rate in beats per minute (bpm) after the execution and imagination of the 2-minute walk test, with a statistically significant difference $\left({ }^{*} p<0.001\right)$. Note that when comparing the heart rate between the moments before the execution and the moments before the imagination of the 2-minute walk test (2MWT), there was no statistical difference, indicating that the participants started from a similar baseline.

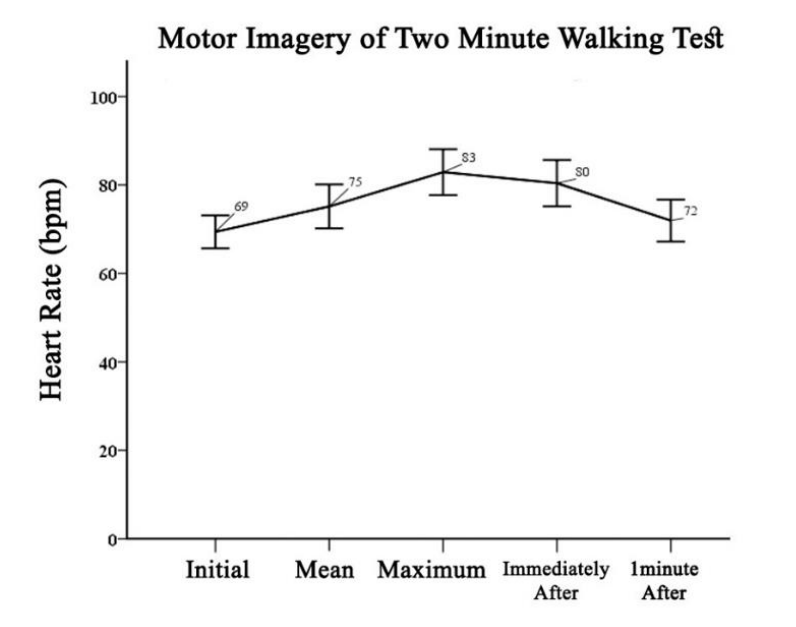

Figure 2 . The line graph shows the heart rate behavior in beats per minute of the participants $(n=20)$ before, during, and after the imagination of the 2-minute walk test. The following data are presented as mean \pm standard deviation: initial heart rate (69.4 \pm 7.56$)$, mean heart rate $(75.1 \pm 10.08)$, maximum heart rate $(82.9 \pm 10.56)$, heart rate immediately after motor imagery of the 2-minute walk test $(80.4 \pm 10.65)$, and heart rate after one $\min (71.9 \pm 9.64)$. 


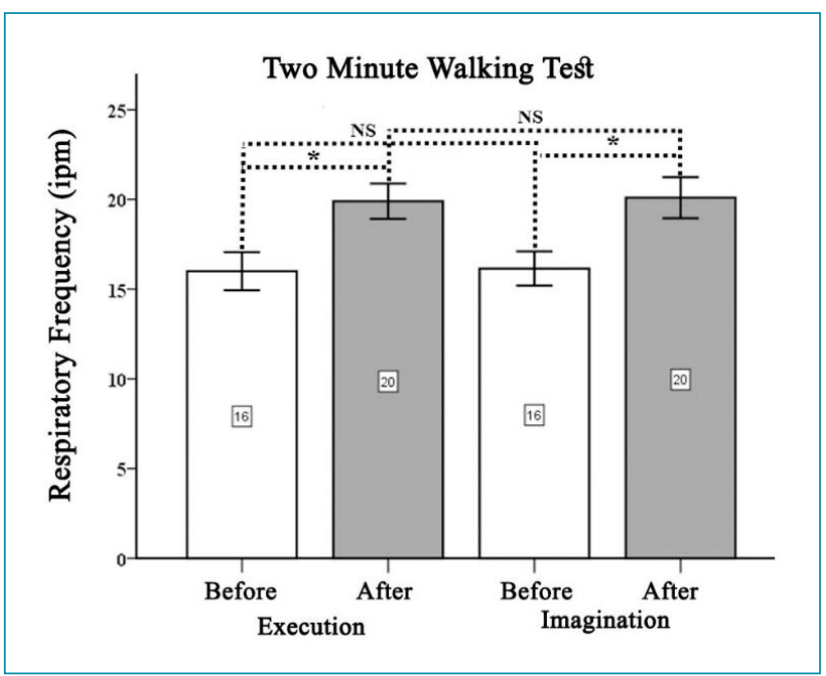

Figure 3 . The participants $(n=20)$ showed an increase in respiratory frequency in incursions per minute (ipm) after the execution and imagination of the 2-minute walk test, with a statistically significant difference $\left({ }^{*} p<0.001\right)$. Note that when comparing respiratory frequency between the moments before the execution and the moments before the imagination, as well as after the execution and after the imagination of the 2-minute walk test (2MWT), there was no statistical difference, indicating similarities.

\section{DISCUSSION}

The object of this study was to investigate the acute effect of kinesthetic MI of the 2MWT on the hemodynamic and cardiopulmonary variables of patients with HF. The main results observed showed that

(1) there was no difference in the number of laps performed and imagined, indicating that the participants actually imagined the test and;

(2) the MI of the 2MWT immediately increased the HR and RF of the participants.

In the context of MI, there are different aspects (temporal and biomechanical) that may have similarities, such as the number of repetitions executed and imagined during a fixed time $e^{4,5}$, as well as the time that an individual requires to walk and imagine that he/she is walking a fixed distance ${ }^{6}$. In this study, it was observed that the number of laps executed and imagined in the 2MWT (biomechanical aspect) did not show statistical difference, indicating that the participants actually imagined the proposed tasks due to the similarities observed. The kinesthetic MI strategy has shown greater modulation in $\mathrm{HR}$ and RF, when compared with the visual MI strategy ${ }^{19}$, justifying its use in this study. Besides that, the high levels of vividness in KVIQ-10 ( $>3$ ) have shown greater modulation using kinesthetic MI when compared with low levels of vividness
$(<2)$ and visual $\mathrm{MI}^{5}$. In this study, high levels of vividness of the movement were observed after the kinesthetic MI of the 2MWT (mean of 3.9), which may partially explain the effects of autonomic modulation observed.

Usually, when an individual plans to start a physical activity (e.g., walking), the areas of preparation and programming of movement are accessed (cerebellum, basal ganglia, reticular formation, ventrolateral bulb, and parieto-frontal area). At this time, the increase in sympathetic influx (cholinergic fibers) and the reciprocal inhibition of parasympathetic activity facilitate the anticipatory cardiopulmonary response, increasing $\mathrm{HR}$ and $\mathrm{RF}^{11,13}$. Similarly, when a healthy individual imagines this same physical activity, areas related to the preparation and programming of movement are also accessed, activating the cardiopulmonary anticipatory mechanisms $\mathrm{s}^{10,20}$ and partially explaining the observed effects. These modulations probably occur due to the change in neuronal activity, mainly in the reticular formation and in the ventrolateral bulb, located in the brain stem (which receives hypothalamic projections), responsible for modulating cardiopulmonary rhythmicity ${ }^{11,13}$.

In this context, MI that is performed consciously is able to induce unconscious neurophysiological changes, when accessing the brain autonomic region responsible for controlling vital signs to keep the system in balance ${ }^{8,19}$. The cardiopulmonary anticipation mechanism can explain the elevations in HR and RF observed in healthy individuals ${ }^{8,10}$, since the brain is able to anticipate the metabolic demands necessary to supply the demand for the exercise milliseconds before its execution ${ }^{21}$. However, until then, these anticipatory cardiopulmonary responses during MI were known only in healthy individuals and/or athletes ${ }^{8-10,22,23}$. In this study, it was observed that, even though patients have HF in class II, these anticipatory mechanisms do not seem to be altered by the pathophysiology of the disease, indicating that this group may benefit from kinesthetic $\mathrm{MI}$ as an adjunctive therapeutic strategy.

In this study, significant increases in $\mathrm{HR}$ and RF were observed immediately after the kinesthetic MI of the 2MWT, corroborating the findings of different studies that observed similar results in healthy individuals when performing the kinesthetic MI of anaerobic exercises in upper ${ }^{19}$ and lower limbs $^{8}$ and aerobics, ${ }^{9,23-25}$ as the gait $\mathrm{MI}^{9,23}$. The MI of fast walking and running at different speeds has shown an increase in SBP. These effects seem to be related to the intensity of the mental effort generated by the participant ${ }^{9}$. The 2MWT consists of an exercise of low physical effort in its execution and mental in its imagination, explaining, in part, the non-modulation of SBP and DBP. Therefore, it seems that MI with a low level of mental effort may be relevant in cardiopulmonary physiotherapy because it does not present a risk of decompensation.

There were no changes in $\mathrm{SpO}_{2}$ after MI of the 2MWT. Similarly, there are also no changes in oxygen consumption during gait $\mathrm{MI}^{8}$, 
indicating that $\mathrm{MI}$ does not cause changes in $\mathrm{O}_{2}$ supply or metabolic expenditure. Recent evidence suggests that kinesthetic MI of breathing movements can control the levels of metabolic expenditure $^{26}$. In addition, there was no acute change in respiratory biomechanics, indicating that MI did not interfere with chest mobility, resistance to expiratory flow, and respiratory muscle strength, as well as in the Borg fatigue and SDS, indicating that there was no patient decompensation after the MI of the 2MWT.

The results of this study point to the possibility of using kinesthetic MI safely, as a strategy in support of the classic cardiorespiratory physiotherapy programs of patients with $\mathrm{HF}$ in functional class I or II. However, the interpretation of these results requires caution for class III or IV, as the effects of MI on these patients are not yet known. Thus, further studies are needed to answer these and other hypotheses that may or may not change the clinical condition of these patients in the long term.

The study presents some limitations:

(1) the small sample size and

(2) the non-quantification of the participants' brain activity (e.g., electroencephalogram) or autonomic activity (e.g., electrocardiogram or echocardiogram) during the MI of the 2MWT.

However, these limitations do not neutralize the innovation of the work due to the correlation between MI and cardiopulmonary variability, even though sophisticated instruments were not used in this study. Despite the limitations described, the study has considerable scientific value, being a pioneer in MI research associated with autonomic functions in patients with HF.

\section{CONCLUSIONS}

The kinesthetic MI of the 2MWT showed an immediate increase in the HR and RF of patients with HF in functional class II, similar to the effects on healthy individuals described in the literature. This indicates that the anticipatory cardiopulmonary response of these patients was modulated immediately by the MI in a safe way, as there were no changes in the perceived effort scales. However, further studies are needed to investigate the effects of MI associated with cardiovascular physiotherapy.

\section{ACKNOWLEDGMENTS}

The authors would like to thank the UNIFESO's Scientific Initiation and Research Program (PICPq) and the entire group of CLIC professionals, for the indication of patients with $\mathrm{HF}$ and, especially, Marli Gomes de Oliveira, for assisting in scheduling participants.

\section{AUTHORS" CONTRIBUTIONS}

NSS: Conceptualization, Data curation, Formal Analysis, Writingoriginal draft, Writing - review \& editing. ACGM: Conceptualization, Data curation, Formal Analysis, Writing - original draft. KMA: Data curation, Writing - original draft. LBO: Conceptualization, Formal Analysis, Writing - review \& editing. RFSA: Conceptualization, Formal Analysis, Writing - review \& editing. MAAL: Writing review \& editing. MAON: Writing - review \& editing. NSMN: Writing - original draft. VHVB: Formal Analysis, Writing - review \& editing. JGS: Writing - review \& editing. ABSF: Conceptualization, Formal Analysis, Writing - review \& editing.

\section{REFERENCES}

1. Cooper LB, Mentz RJ, Sun JL, Schulte PJ, Fleg JL, Cooper LS, et al. Psychosocial factors, exercise adherence, and outcomes in heart failure patients: insights from heart failure: a controlled trial investigating outcomes of exercise training (HF-ACTION). Circ Heart Fail. 2015;8(6):1044-51. https://doi.org/10.1161/ CIRCHEARTFAILURE.115.002327

2. Bennett JA, Riegel B, Bittner $V$, Nichols J. Validity and reliability of the NYHA classes for measuring research outcomes in patients with cardiac disease. Heart Lung. 2002;31(4):262-70. https://doi.org/10.1067/mhl.2002.124554

3. Lavie CJ, Berra K, Arena R. Formal cardiac rehabilitation and exercise training programs in heart failure: evidence for substantial clinical benefits. J Cardiopulm Rehabil Prev. 2013;33(4):20911. https://doi.org/10.1097/HCR.0b013e31829f95c9

4. Souza NS, Martins A, Ferreira C, Motizuki Y, Machado C, Orsini $M$, et al. Effect of cervical kinesthetic motor imagery on postural control of healthy young adults with fear of falling. J Funct Morphol Kinesiol. 2017;2(2):21. https://doi. org/10.3390/jfmk2020021
5. Lemos T, Souza NS, Horsczaruk CHR, Nogueira-Campos AA, Oliveira LAS, Vargas CD, et al. Motor imagery modulation of body sway is task-dependent and relies on imagery ability. Front Hum Neurosci. 2014;8:290. https://doi.org/10.3389/ fnhum.2014.00290

6. Decety J, Jeannerod M, Prablanc $C$. The timing of mentally represented actions. Behav Brain Res. 1989;34(1-2):35-42. https://doi.org/10.1016/s0166-4328(89)80088-9

7. Ruby P, Decety J. Effect of subjective perspective taking during simulation of action: a PET investigation of agency. Nat Neurosci. 2001;4(5):546-50. https://doi.org/10.1038/87510

8. Decety J, Jeannerod M, Durozard D, Baverel G. Central activation of autonomic effectors during mental simulation of motor actions in man. J Physiol. 1993;461(1):549-63. https://doi.org/10.1113/ jphysiol.1993.sp019528

9. Decety J, Jeannerod M, Germain M, Pastene J. Vegetative response during imagined movement is proportional to mental effort. Behav Brain Res. 1991;42(1):1-5. https://doi. org/10.1016/s0166-4328(05)80033-6 
10. Oishi K, Kasai T, Maeshima T. Autonomic response specificity during motor imagery. J Physiol Anthropol Appl Human Sci. 2000;19(6):255-61. https://doi.org/10.2114/jpa.19.255

11. Javorka M, Czippelova B, Turianikova Z, Lazarova Z, Tonhajzerova I, Faes L. Causal analysis of short-term cardiovascular variability: state-dependent contribution of feedback and feedforward mechanisms. Med Biol Eng Comput. 2017;55(2):179-90. https://doi.org/10.1007/s11517-016-1492-y

12. Decety J, Perani D, Jeannerod M, Bettinardi V, Tadary B, Woods $R$, et al. Mapping motor representations with positron emission tomography. Nature. 1994;371(6498):600-2. https:// doi.org/10.1038/371600a0

13. Mcardle WD., Katch FI, Katch VL. Exercise physiology: nutrition, energy, and human performance. 8th ed. Philadelphia: Wolters Kluwer Health; 2014. 1028 p.

14. Souza NS, Martins ACG, Samary CS, Leite MAA, Bastos VH. The use of motor imagery in modulating cardiopulmonary activity: future perspectives. Acta Neuropsychol. 2019 [cited on Jul. 26, 2019];1(1):180102. Available from: https://www. researchgate.net/publication/338867686_Citation_Souza_NS_ The_Use_of_Motor_Imagery_in_Modulating_Cardiopulmonary_ Activity_Future_Perspectives

15. Bertolucci PHF, Brucki SMD, Campacci SR, Juliano Y. O miniexame do estado mental em uma população geral: impacto da escolaridade. Arq Neuropsiquiatr. 1994;52(1):1-7. PMID: 8002795.

16. Coutinho-Myrrha MA, Dias RC, Fernandes AA, Araújo CG, Hlatky MA, Pereira DG, et al. Duke activity status index for cardiovascular diseases: validation of the portuguese translation. Arq Bras Cardiol. 2014;102(4):383-90. https:// doi.org/10.5935/abc.20140031

17. Malouin F, Richards CL, Jackson PL, Lafleur MF, Durand A, Doyon J. The Kinesthetic and Visual Imagery Questionnaire (KVIQ) for assessing motor imagery in persons with physical disabilities: a reliability and construct validity study. J Neurol Phys Ther. 2007;31(1):20-9. https://doi.org/10.1097/01.npt.0000260567.24122.64

18. Brooks D, Parsons J, Tran D, Jeng B, Gorczyca B, Newton J, et al. The two-minute walk test as a measure of functional capacity in cardiac surgery patients. Arch Phys Med Rehabil. 2004;85(9):1525-30. https://doi.org/10.1016/j. apmr.2004.01.023

19. Wang $Y$, Morgan WP. The effect of imagery perspectives on the psychophysiological responses to imagined exercise. Behav Brain Res. 1992;52(2):167-74. https://doi.org/10.1016/ s0166-4328(05)80227-x

20. Decety J, Perani D, Jeannerod M, Bettinardi V, Tadary B, Woods $\mathrm{R}$, et al. Mapping motor representations with positron emission tomography. Nature. 1994;371(6498):600-2. https:// doi.org/10.1038/371600a0

21. Calomeni MR, Almeida MWS, Arêas Neto NT, Silva VF. Variação da frequência cardíaca durante uma sessão de estimulação cortical e imagética. Fit Perf J. 2009;8(1):5-8. https://doi. org/10.3900/fpj.8.1.5.p

22. Decety J, Kawashima R, Gulyás B, Roland PE. Preparation for reaching: a PET study of the participating structures in the human brain. Neuroreport. 1992;3(9):761-4. PMID: 1421133

23. Wuyam B, Moosavi SH, Decety J, Adams L, Lansing RW, Guz A. Imagination of dynamic exercise produced ventilatory responses which were more apparent in competitive sportsmen. J Physiol. 1995;482(Pt 3):713-24. https://doi.org/10.1113/ jphysiol.1995.sp020554

24. Oishi K, Kimura M, Yasukawa M, Yoneda T, Maeshima T. Amplitude reduction of $\mathrm{H}$-reflex during mental movement simulation in elite athletes. Behav Brain Res. 1994;62(1):5561. https://doi.org/10.1016/0166-4328(94)90037-x

25. Guillot A, Collet C, Dittmar A. Influence of environmental context on motor imagery quality: an autonomic nervous system study. Biol Sport. 2005 [cited on Jan. 6, 2005];22(3):215-26. Available from: https://www.researchgate.net/publication/234051475_ Influence_of_environmental_context_on_motor_imagery_ quality

26. Kanthack TFD, Guillot A, Saboul D, Debarnot U, Di Rienzo F. Breathing with the mind: effects of motor imagery on breathhold performance. Physiol Behav. 2019;208:112583. https:// doi.org/10.1016/j.physbeh.2019.112583 